Signal \& Image Processing : An International Journal (SIPIJ) Vol.6, No.4, August 2015

\title{
ENERGY MINIMIZATION-BASED SPATIALLY CONSTRAINED MIXTURE MODEL AND ITS APPLICATION TO IMAGE SEGMENTATION
}

\author{
Zhiyong Xiao, Yunhao Yuan, Jianjun Liu and Jinlong Yang \\ School of Things Engineering, also with Key Laboratory of \\ Advanced Process Control for Light Industry (Ministry of Education), \\ Jiangnan University, Wuxi 214122, China.
}

\begin{abstract}
A novel method is proposed for image segmentation based on probabilistic field theory. This model assumes that the whole pixels of an image and some unknown parameters form a field. According to this model, the pixel labels are generated by a compound function of the field. The main novelty of this model is it consider the features of the pixels and the interdependent among the pixels. The parameters are generated by a novel spatially variant mixture model and estimated by expectation-maximization (EM)based algorithm. Thus, we simultaneously impose the spatial smoothness on the prior knowledge. Numerical experiments are presented where the proposed method and other mixture model-based methods were tested on synthetic and real world images. These experimental results demonstrate that our algorithm achieves competitive performance compared to other methods.
\end{abstract}

\section{KEYWORDS}

Probabilistic field theory, spatial constraints, parameter estimate, expectation-maximization(EM) algorithm, mixture model, image segmentation.

\section{INTRODUCTION}

Image segmentation plays an important step in image processing and computer vision. The task of image segmentation is to classify image pixels based on the coherence of certain features such as intensity, color, texture, motion, location. Many methods have been previously proposed for image segmentation [1], [2]. These methods can be classified into two groups: contour-based approaches and region-based approaches.

The aim of the contour-based approaches is to find the boundaries of objects in an image. Many contour-based segmentation algorithms have been developed, such as "snakes" or active contours [3], GVF (gradient vector flow) [4], VFC (vector field convolution) [5], Sobolev gradient [6].

Region-based approaches classify an image into multiple consistent classes. The main research directions are focused on graph cut approaches [7], mean shift algorithm [8] and clustering based 
Signal \& Image Processing : An International Journal (SIPIJ) Vol.6, No.4, August 2015

methods [9]. In this paper, we will focus our attention on clustering methods for image segmentation.

The mixture model (MM) [10], [11] is the most commonly used model for clustering. In this approach, the pixels are viewed as coming from a mixture of probability distributions, each representing a different component. The parameters of the probability distributions can be estimated very efficiently through maximum likelihood (ML) using the expectation-maximization (EM) algorithm [12], [13]. When the parameters have been estimated, each pixel can be assigned using the maximum a posteriori (MAP) rule.

The main advantage of the $\mathrm{MM}$ is that it has simple mathematical form and it is easy to implement. Thus, it has been used successfully in a number of applications [14], [15]. A drawback of the MM is that it doesn't use the spatial information about the data [16]. In order to overcome this drawback, the spatial information need to be incorporated into the model and several approaches were proposed.

Markov random field (MRF) [17], [18], [19] theory provides a convenient and consistent way to account for spatial dependencies between image pixels. The spatially variant finite mixture model (SVFMM) [16], [20], [21] assumes that the prior distribution form a Markov field. The prior distribution of the SVFMM depends on then neighboring pixels and their corresponding parameters. Thus, these models work well in medical images for segmentation [22]. However, the drawback is that they are computationally expensive to estimate parameters. Various approximations have been introduced to tackle this problem. The gradient projection algorithm [16] was proposed to implement the M-step of the EM algorithm. A closed-form update equation followed by an efficient projection method [20] is used to estimate the parameters.

In [23], a new family prior distributions was proposed for SVFMM. The proposed prior probability is based on Gauss-Markov random field, which control the degree of smoothness for each cluster. The parameters can be estimated in closed form via the MAP estimation using the EM algorithm. In [24], the Dirichlet Compound Multinomial-based Spatially Variant Finite Mixture Model (DCM-SVFMM) was proposed. This model exploits the Dirichlet compound multinomial probability to model the probabilities of class labels and the Gauss-Markov random field to model the spatial prior.

Another approach to take the commonality of location into account is extension of mixture model [25]. This method is quite similar to the mixture model and easy to implement. It introduces a novel prior distribution which acts like a mean filter to incorporate the spatial relationships between neighboring pixels. In [21], a spatially constrained generative model was proposed for segmentation. It assumes the prior distributions share similar parameters for neighboring pixels and introduces a pseudo-likelihood quantity to measure the similarity between neighboring pixels priors.

In this paper, we introduce a probabilistic field theory-based model for image segmentation. This model assumes that the whole given image and some unknown parameters construct a field and the labels of image pixels are functions of the field. Therefore, the mixture model-based segmentation can be considered as a special case of the proposed framework. Furthermore, in order to incorporate the spatial relationships between the neighboring pixels, we propose a novel spatially variant mixture model to estimate the parameters. 
Signal \& Image Processing : An International Journal (SIPIJ) Vol.6, No.4, August 2015

We also propose a novel approach to properly incorporate the spatial relationships between the neighboring pixels into the priors. The proposed probabilistic field theory-based model account for the spatially local interaction. Numerical experiments are presented to assess the performance of the proposed model both with simulated data and real natural images. We compare the results of our proposed method to other methods both visually and quantitatively.

The remainder of this paper is organized as follows. In Section II, we present a brief introduction of the image segmentation problem and some mixture model-based methods. We describe the details of the proposed model and the algorithm in Section III and Section IV, respectively. The experimental results are shown in Section V.

\section{A REviEW OF MiXTURe Model-BASEd Methods For IMAGE SEGMENTATION}

In this section, we discuss four commonly used mixture model-based methods for image segmentation. The first group is the standard mixture model in which each pixel is considered independent of its neighbors. In order to incorporate the spatial information into the mixture model, a spatially variant finite mixture model (SVFMM) is proposed, which assumes the prior distributions that generate the pixel labels form a Markov random field. Third one is an extension of standard mixture model. The last group is an extension of the SVFMM.

Firstly we define the image multi-class segmentation problem. Given an image $\mathrm{X}$ which has $\mathrm{N}$ pixels, we suppose it could be partitioned into $\mathrm{K}$ classes $\mathrm{U}_{\mathrm{k}} ; \mathrm{k}=1,2, \ldots, \mathrm{K}$ with the following properties

$$
X=\cup_{k=1}^{K} U_{k}, U_{s} \cap U_{t}=\varnothing \text { if } s \neq t
$$

Then we provide a brief description of the multi-class image labeling problems. Consider a set of random variables $E=\left\{e_{1}, e_{2}, \ldots, e_{N}\right\}$. The random field is defined on the image $X$, where each pixel $i$ is associated with a random variable $e_{i}$. Each variable $e_{i}=\left(e_{i l}, e_{i 2}, \ldots, e_{i k}\right)$ represents a region assignment for pixel $i$, where eik is either 0 or $1 . P\left(e_{i k}=1 \mid x_{i}\right)$ for all classes, it is assigned to the class with the largest posterior probability

$$
x_{i} \in U_{k}: \quad \text { IF } \quad k=\arg \max _{t} P\left(e_{i t}=1 \mid x_{i}\right)
$$

\section{A. Mixture Model}

The mixture model [11] assumes a common prior distribution $\pi$ which is a discrete distribution with $\mathrm{K}$ states, whose parameters $\pi_{\mathrm{k},} k=1,2, \ldots, K$ are unknown, and holds:

$$
P\left(e_{i k}=1\right)=\pi_{k}
$$

where we see that the prior $\pi_{\mathrm{k}}$ has no dependence on pixel index $i$ and the prior distribution satisfies the constraints

$$
0 \leq \pi_{k} \leq 1 \text { and } \sum_{k=1}^{K} \pi_{k}=1
$$


Signal \& Image Processing : An International Journal (SIPIJ) Vol.6, No.4, August 2015

The mixture model assumes that the density function at a pixel observation $x_{i}$ is given by

$$
P\left(x_{i}\right)=\sum_{k=1}^{K} P\left(e_{i k}=1\right) P\left(x_{i} \mid e_{i k}=1, \theta\right)
$$

where $P\left(x_{i} \mid e_{i k}=1, \theta\right)$ is a density conditional on the class label $k$, i.e.:

$$
P\left(x_{i} \mid e_{i k}=1, \theta\right)=P\left(x_{i} \mid \theta_{k}\right)
$$

where $\theta_{k}$ is the parameter of the kth component distribution.

After the mixture model has been fitted, the next step is to estimate the parameters using the maximum likelihood approach. The log-likelihood function of all observations $X=\left\{x_{1}, x_{2}, \ldots, x_{N}\right\}$ for the $N$ pixels is given by

$$
\begin{aligned}
L(\theta, \pi) & =\sum_{i=1}^{N} \log \sum_{k=1}^{K} P\left(e_{i k}=1\right) P\left(x_{i} \mid e_{i k}=1, \theta\right) \\
& =\sum_{i=1}^{N} \log \sum_{k=1}^{K} \pi_{k} P\left(x_{i} \mid \theta_{k}\right)
\end{aligned}
$$

The log-likelihood function is considered as a function of the parameters $\theta$ where the data $X$ is fixed. The parameters are estimated by the EM algorithm [12], [13].

\section{B. Spatially Variant Finite Mixture Model}

In the mixture model, the pixels are considered independent. In order to account for the spatial dependence between image pixels, the spatially variant finite mixture model(SVFMM) [16], [20] was proposed. The density function at an observation $x_{i}$ is given by

$$
\begin{aligned}
P\left(x_{i}\right) & =\sum_{k=1}^{K} P\left(e_{i k}=1\right) P\left(x_{i} \mid e_{i k}=1, \theta\right) \\
& =\sum_{k=1}^{K} \pi_{i k} P\left(x_{i} \mid \theta_{k}\right)
\end{aligned}
$$

where the parameter $\pi_{\mathrm{ik}}$ is the prior distribution of the pixel $x_{i}$ belonging to the $k t h$ class, which satisfies the constraints:

$$
0 \leq \pi_{i k} \leq 1 \text { and } \sum_{k=1}^{K} \pi_{i k}=1
$$

The SVFMM assumes that the prior distribution $\vdash$ that generate the pixel labels form a Markov random field. The random field of the prior distribution is defined [16]:

$$
P(\pi)=\frac{1}{Z} \exp (-\beta U(\pi))
$$


Signal \& Image Processing : An International Journal (SIPIJ) Vol.6, No.4, August 2015

where $\mathrm{Z}$ is a normalizing constant called the partition function, $\beta$ is a regularization constant. The function $\mathrm{U}(\pi)$ denotes the clique potential function on $\pi$ which considers the spatial interaction information and can be computed as follows:

$$
U(\pi)=\sum_{i=1}^{N} \sum_{j \in N(i)} \sum_{k=1}^{K}\left(\pi_{i k}-\pi_{j k}\right)^{2}
$$

where $N(i)$ is the neighborhood of $i$.

The log-likelihood function becomes:

$$
L(\theta, \pi)=\sum_{i=1}^{N} \log \sum_{k=1}^{K} \pi_{i k} P\left(x_{i} \mid \theta_{k}\right)-\beta \sum_{k=1}^{K} \sum_{i=1}^{N} \sum_{j \in N(i)}\left(\pi_{i k}-\pi_{j k}\right)^{2}
$$

The above log-likelihood function is more complex than the standard mixture model. Since there are $\mathrm{N}$ different $\pi_{\mathrm{i}}$ distributions, one for each pixel $i$. The M-step of EM algorithm cannot be applied directly to the prior distribution $\pi_{\mathrm{i} .}$. In [16], [20] and [26], a large amount of computational effort goes in the estimation of the $\pi_{\mathrm{i}}$ priors in the M-step.

In standard mixture model, only the intensity value of pixel determines the segmented result. While the segmented result of SVFMM is based on intensity value and the spatial information.

\section{A Class-Adaptive Spatially Finite Mixture Model}

In this section, an extension of Spatially Variant Finite Mixture Model [23] is presented. In this model, the prior probability is considered to be based on Gauss-Markov random field. The novel prior probability allows their parameters to be estimated in closed form using the EM methodology [16], [20] and [26]. The Gauss-Markov random field prior probability for $\pi$ in (11) is given by

$$
P(\pi)=\prod_{k=1}^{K} \beta_{k}^{-N} \exp \left(-\frac{1}{2} \frac{\sum_{i=1}^{N} \sum_{j \in N(i)}\left(\pi_{i k}-\pi_{j k}\right)^{2}}{\beta_{k}^{2}}\right)
$$

where $N(i)$ is the neighborhood for the pixel $i$, the parameters $\beta_{k}^{2}$ capture the spatial smoothness of class $k$. In this Class-adaptive spatially mixture model (CA-SVFMM), the prior can capture the smoothness of each class in different degrees and adapts better to the data.

The predictor of the prior probability $\pi_{\mathrm{ik}}$ is defined by the mean of its spatial neighbors as

$$
\hat{\pi}_{i k}=\frac{1}{N(i)} \sum_{j \in N(i)} \pi_{j k}
$$

where $N(i)$ is the number of pixels in the neighborhood.

The prior statistical assumption is based on 
Signal \& Image Processing : An International Journal (SIPIJ) Vol.6, No.4, August 2015

$$
P\left(N(i) \epsilon_{i k}\right)=N\left(0, \beta^{2}\right)
$$

where $\epsilon_{i k}=\pi_{i k}-\hat{\pi}_{i k}$ is the prediction error, $\mathrm{N}\left(0, \beta^{2}\right)$ is Gaussian random variables with zero mean and variance $\beta^{2}$.

Moreover, the parameter $\beta$ of the prior probability can also capture the smoothness in all directions. In [23], the authors introduce parameters $\beta_{k d}^{2}$, which expresses not only the class variance for class $\mathrm{k}$ but also the variance within class $\mathrm{k}$ at a certain spatial direction $\mathrm{d}$. In this case, the prior is given by

$$
P(\pi)=\prod_{d=1}^{D} \prod_{k=1}^{K} \beta_{k d}^{-N} \exp \left(-\frac{1}{2} \frac{\sum_{i=1}^{N} \sum_{j \in N(i)}\left(\pi_{i k}-\pi_{j k}\right)^{2}}{\beta_{k d}^{2}}\right)
$$

Using the Gauss-Markov random field-based prior probability, the EM algorithm [16], [20] and [26] is utilized for estimating the parameters. The details of the algorithm is presented in [23].

\section{PROPOSED METHOD}

In this section, we introduce a probabilistic field theory-based model, which assumes the whole image pixels and some unknown parameters construct a field. The mixture model-based methods can be considered as special approaches for estimating parameters. Furthermore, we propose a new spatially constrained mixture model for parameter estimation. In order to consider the interaction between the posterior probabilities, we also proposed a spatially constrained probabilistic field theory-based model for image segmentation.

\section{A. Probabilistic Field Theory-based Model}

Field theory [27], [28] is a classic framework for the analysis of social behavior. Employing the conventions of mathematics, the Lewin's equation represents the idea that human behavior is related both to one's personal characteristics and to the environment

$$
\mathbf{B}=f(\mathbf{P}, \mathbf{E})
$$

where $\mathbf{B}$ stands for the individual's publicly observable behavior, $\mathbf{P}$ stands for all the causal factors of the individual person, and $\mathbf{E}$ stands for all the causal factors of the environment. This equation is graphically shown in Fig.1(a).

We propose an extension of the Lewin's equation using the probability theory.

$$
\begin{array}{r}
P(\mathbf{B})=f(\mathbf{P}, \mathbf{E}) \\
\mathbf{B}=g(P(\mathbf{B}))
\end{array}
$$


Signal \& Image Processing : An International Journal (SIPIJ) Vol.6, No.4, August 2015

where $P(\mathbf{B})$ is the probability of the behavior, and the behavior is a function of the probability $P(\mathbf{B})$, as shown in Fig.1(b).

The above image segmentation problem in (1) can be considered as a process of labeling:

$$
x_{i} \in U_{k} \Longleftrightarrow e_{i k}=1
$$

For the image labeling problem, the random variable $e_{i}=\left(e_{i 1}, e_{i 2}, \ldots, e_{i k}\right)$ can be considered as the behavior of pixel $i$. We define a set of parameters $\theta$ as the environment factors. So the probabilistic field theory for image segmentation is:

$$
\begin{gathered}
P\left(e_{i}\right)=f\left(x_{i}, \Theta\right) \\
e_{i}=g\left(P\left(e_{i}\right)\right)
\end{gathered}
$$

where $\mathrm{P}\left(\mathrm{e}_{\mathrm{i}}\right)=\left(\mathrm{P}\left(\mathrm{e}_{\mathrm{i} 1}=1 \mid \mathrm{x}_{\mathrm{i}}\right), \ldots, \mathrm{P}\left(\mathrm{e}_{\mathrm{ik}}=1 \mid \mathrm{x}_{\mathrm{i}}\right)\right)$ is a vector, which presents the probabilities of the labels for the pixel $i$. $\mathrm{P}\left(\mathrm{e}_{\mathrm{ik}}=1 \mid \mathrm{x}_{\mathrm{i}}\right)$ is the probability of the pixel $i$ belonging to the class $\mathrm{k} . \mathrm{x}_{\mathrm{i}}$ is the observed value of pixel $i$. It is graphically shown in Fig.1(c).

In our method, we define function $f$ using the Bayesian rule:

$$
P\left(e_{i k}=1 \mid x_{i}\right)=\frac{P\left(e_{i k}=1\right) P\left(x_{i} \mid e_{i k}=1, \theta\right)}{\sum_{d=1}^{K} P\left(e_{i d}=1\right) P\left(x_{i} \mid e_{i d}=1, \theta\right)}
$$

And for each pixel $i$, we define function $g$ using the maximum a posterior rule(MAP) to assign it:

$$
e_{i k}= \begin{cases}1 \quad \text { if } k=\arg \max _{t} P\left(e_{i t}=1 \mid x_{i}\right) \\ 0 \quad \text { if otherwise }\end{cases}
$$

The probabilistic field theory-based model is complete only if its parameters are determined. Thus, before we use our model to segment an image, we need to estimate the parameters. The mixture modelbased methods are commonly used approaches for parameters estimation. In this paper, we present a new spatially constrained mixture model to estimate the parameters.

\section{B. A New Spatially Constrained Mixture Model for Parameter Estimation}

We propose a new family of smoothness priors to take into account the spatial statistical information.

The weight function is defined as in the extension of the mixture model [25]

$$
\xi_{k}\left(x_{i}\right)=\exp \left(-\frac{\left(x_{i}-c_{k}\right)^{2}}{2 b_{k}^{2}}\right)
$$


Signal \& Image Processing : An International Journal (SIPIJ) Vol.6, No.4, August 2015

We denote a new prior distribution

$$
\pi_{i k}=\sum_{j \in N(i)} \frac{h(i, j)}{Z_{i}} \xi_{k}\left(x_{j}\right)
$$

where

$$
Z_{i}=\sum_{j \in N(i)} h(i, j)
$$
distance) between pixel $i$ and $j$. In this paper, the geometric closeness $h$ is Gaussian function of the magnitude of the relative position vector of pixel $j$ from pixel $i$, $\left\|u_{i}-u_{j}\right\|$.

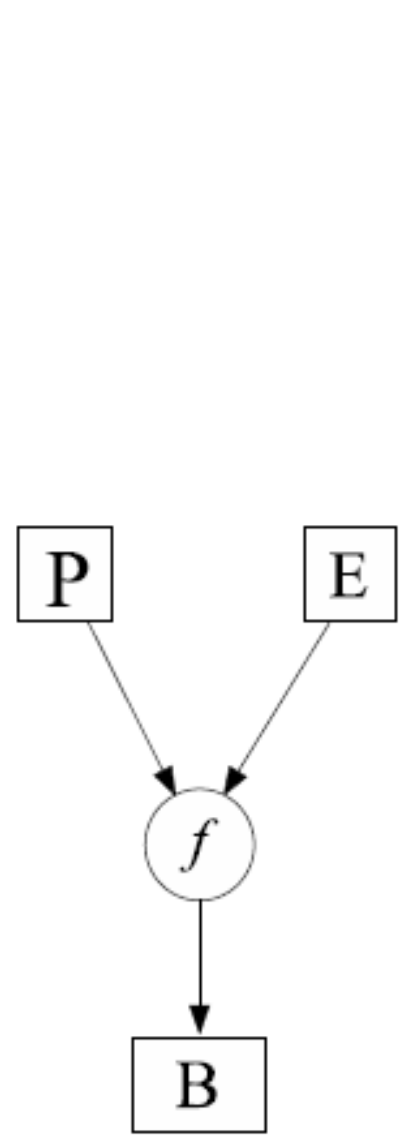

(a)

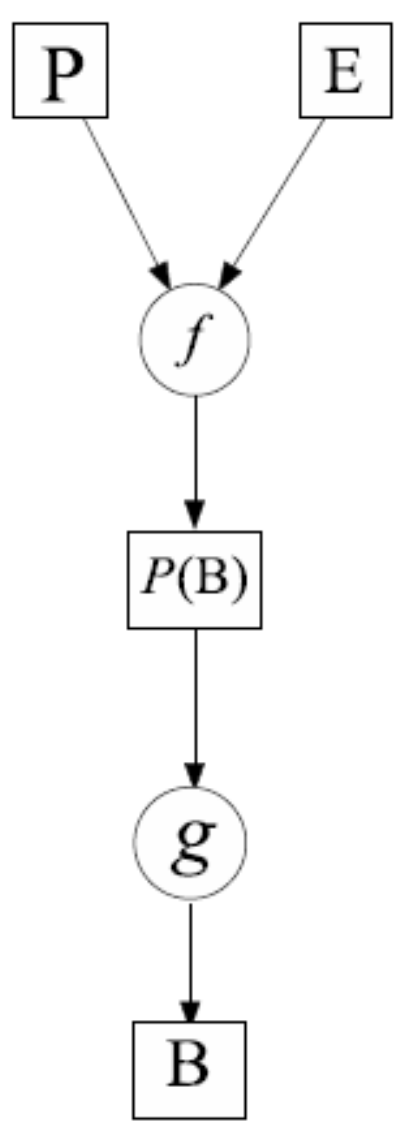

(b)

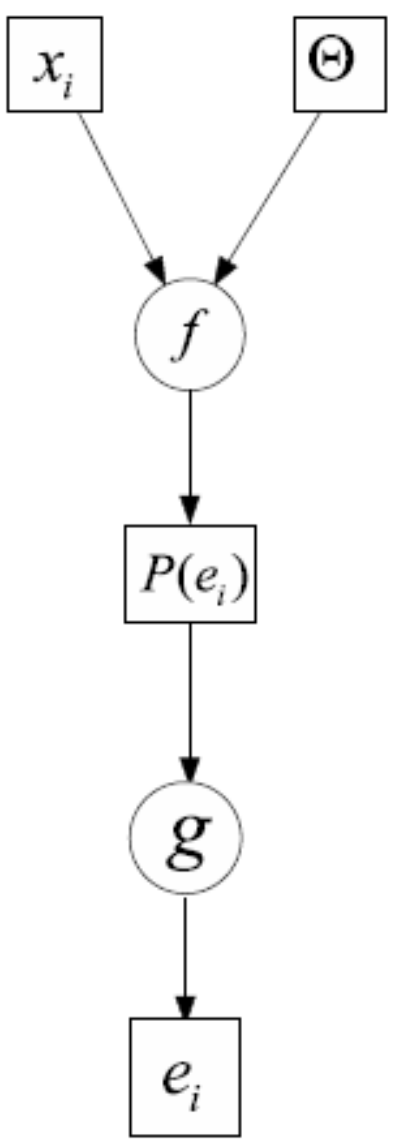

(c)

Fig. 1. (a) Field Theory for analysis of social behavior. (b) Probabilistic Field Theory for analysis of social behavior. (c) Probabilistic Field Theory for image segmentation.

The geometric closeness function is given as a decreasing function $h$ when the distance $\left\|u_{i}-u_{j}\right\|$ increases: 
Signal \& Image Processing : An International Journal (SIPIJ) Vol.6, No.4, August 2015

$$
h(i, j)=\exp \left(\frac{-\left\|u_{i}-u_{j}\right\|^{2}}{2 \sigma_{g}^{2}}\right)
$$

where $\sigma_{\mathrm{g}}$ is parameter, which defines the desired structural locality between neighboring pixels, $u_{i}$ is the location of the pixel $i$.

The density function of this mixture model at an observation $x_{i}$ is given by

$$
\begin{aligned}
P\left(x_{i}\right) & =\sum_{k=1}^{K} \pi_{i k} P\left(x_{i} \mid \theta_{k}\right) \\
& =\sum_{k=1}^{K} \sum_{j \in N(i)} \frac{h(i, j)}{Z_{i}} \xi_{k}\left(x_{j}\right) P\left(x_{i} \mid \theta_{k}\right)
\end{aligned}
$$

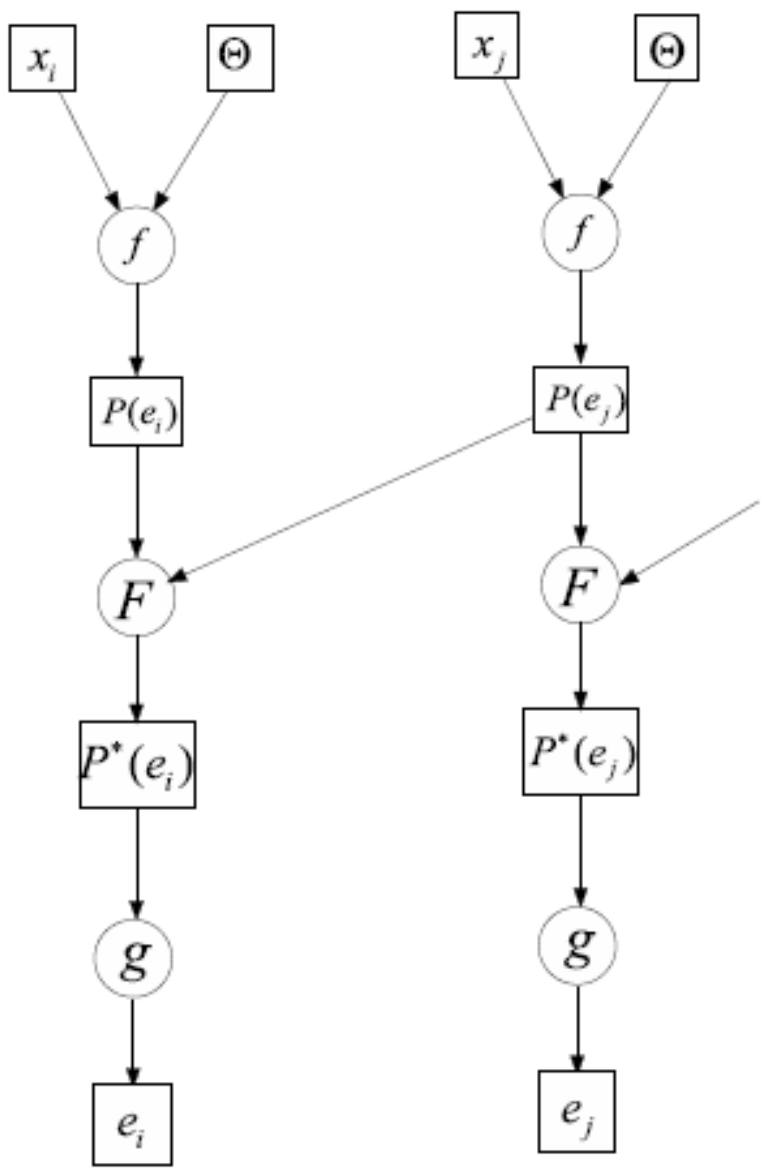

Fig. 2. A Spatially Constrained Probabilistic Field Theory for image segmentation.

Using the above proposed spatially constrained mixture model, the log-likelihood function can be computed as follow 
Signal \& Image Processing : An International Journal (SIPIJ) Vol.6, No.4, August 2015

$$
L(\theta, \pi)=\sum_{i=1}^{N} \log \sum_{k=1}^{K} \sum_{j \in N(i)} \frac{h(i, j)}{Z_{i}} \xi_{k}\left(x_{i}\right) P\left(x_{i} \mid \theta_{k}\right)
$$

The parameters are estimated by maximizing the above likelihood L. The algorithm will be presented in the next section.

\section{A Spatially Constrained Probabilistic Field Theory-based Model for Image Segmentation}

The above proposed mixture model utilizes a novel approach to incorporate the spatial relationships between neighboring pixels into the prior distribution. However, in the probabilistic field theory-based model for image segmentation, we assume that there is no interaction among the posterior probabilities for different pixels. In fact, the posterior probability of one pixel can be influenced by the posterior probabilities of its neighbor pixels. Thus, we propose a spatially constrained probabilistic field theory based model for image segmentation. In the proposed model, to take the interactions of the posterior distribution into account, we add a function $F$ between function $f$ in (16) and function $\mathrm{g}$ in (16) to incorporate the dependence of observed data (image pixels).

Motivated by the above we introduce a new function $F$, which model the interaction over the probability distribution of the assignment

$$
P^{*}\left(e_{i k}=1 \mid x_{i}\right)=F\left(P\left(e_{j k}=1 \mid x_{j}\right), j \in N(i)\right)
$$

where $N(i)$ denotes a set of pixels adjacent to pixel i with respect to a neighborhood system.

The function $\mathrm{F}$ is defined as

$$
F\left(P\left(e_{j k}=1 \mid x_{j}\right), j \in N(i)\right)=\sum_{j \in N(i)} \frac{h(i, j)}{Z_{i}} P\left(e_{j k}=1 \mid x_{j}\right)
$$

where $\mathrm{Z}_{\mathrm{i}}$ is the normalizing factor and $h(i, j)$ is the function measuring the geometric closeness, which are defined in (21).

The proposed model for image segmentation is present as follows:

$$
\begin{aligned}
P\left(e_{i}\right) & =f\left(x_{i}, \Theta\right) \\
P^{*}\left(e_{i}\right) & =F\left(P\left(e_{i}\right)\right) \\
e_{i} & =g\left(P^{*}\left(e_{i}\right)\right)
\end{aligned}
$$

The model is graphically shown in Fig.2. Compare to the Fig.1(c), it is easy to see that the difference between the probabilistic field theory-based model and the proposed model is the function F. The new posterior probability of the proposed model acts like a mean filter. For that reason, the image segmentation result obtained by employing the proposed model is robust with respect to noise. 
Signal \& Image Processing : An International Journal (SIPIJ) Vol.6, No.4, August 2015

When the parameters have been estimated using the proposed spatially constrained mixture model, the above spatially constrained probabilistic field theory-based model for image segmentation contains three steps:

Step 1:

$$
P\left(e_{i k}=1 \mid x_{i}\right)=\frac{\pi_{i k} P\left(x_{i} \mid \theta_{k}\right)}{\sum_{d=1}^{K} \pi_{i d} P\left(x_{i} \mid \theta_{d}\right)}
$$

Step 2:

$$
P^{*}\left(e_{i k}=1 \mid x_{i}\right)=\sum_{j \in N(i)} \frac{h(i, j)}{Z_{i}} P\left(e_{j k}=1 \mid x_{j}\right)
$$

Step 3 :

$$
e_{i k}= \begin{cases}1 & \text { if } k=\arg \max _{t} P^{*}\left(e_{i t}=1 \mid x_{i}\right) \\ 0 & \text { if otherwise }\end{cases}
$$

\section{ALgORITHM}

In this section, we first adjust the parameters $\Theta=(\theta, \pi)$ to maximize the likelihood function $L$ in (23) using a modified EM algorithm. Then we compute the function $f, \mathrm{~F}$ and $g$ using the estimated parameters.

\section{A. Parameter Estimation Algorithm}

In general, we assume that the probability distribution of the mixture model is Gaussian distribution, which is given by:

$$
P\left(x_{i} \mid \theta_{k}\right)=\frac{1}{\sqrt{2 \pi \sigma_{k}^{2}}} \exp \left(-\frac{\left(x_{i}-\mu_{k}\right)^{2}}{2 \sigma_{k}^{2}}\right)
$$

In this paper, we use the $3 \times 3$ window. Thus, the free parameter $\sigma_{g}^{2}$ of the geometric function $h$ is set to 12 and the normalizing factor $\mathrm{Z}_{\mathrm{i}}$ is constant and set to 8.52, $\forall i$.

We use K-means algorithm to initialize the mean $\mu_{k}^{0}$, and covariance $\sigma_{k}^{0}$, for $\mathrm{k}=1,2, \ldots, \mathrm{K}$. For the $m t h$ iteration, the proposed algorithm can be summarized as follows.

Step 1: Select $c_{k}^{m}=\mu_{k}^{m}$, and $b_{k}^{m}=\sigma_{k}^{m}$, for $k=1,2, \ldots, K$.

Step 2: Calculate the weight function $\xi_{k}^{m}\left(x_{i}\right)$ from (19) and the prior probability $\pi_{i k}^{m}$ from (20).

Step 3(E-Step): Calculate posterior probabilities: 
Signal \& Image Processing : An International Journal (SIPIJ) Vol.6, No.4, August 2015

$$
P\left(e_{i k}^{m}=1 \mid x_{i}\right)=\frac{\pi_{i k}^{m} P\left(x_{i} \mid \theta_{k}^{m}\right)}{\sum_{l=1}^{K} \pi_{i k}^{m} P\left(x_{i} \mid \theta_{k}^{m}\right)}
$$

for $i=1,2, \ldots N ; k=1,2 \ldots K$.

Step 4(M-Step): Update the parameters $\theta^{m+1}$

$$
\begin{aligned}
\mu_{k}^{m+1}= & \frac{\sum_{i=1}^{N} P\left(e_{i k}^{m}=1 \mid x_{i}\right) x_{i}}{\sum_{i=1}^{N} P\left(e_{i k}^{m}=1 \mid x_{i}\right)} \\
\sigma_{k}^{m+1}= & \frac{\sum_{i=1}^{N} P\left(e_{i k}^{m}=1 \mid x_{i}\right)\left(x_{i}-\mu_{k}^{m+1}\right)^{2}}{\sum_{i=1}^{N} P\left(e_{i k}^{m}=1 \mid x_{i}\right)}
\end{aligned}
$$

for all the $i=1,2, \ldots N ; k=1,2, \ldots K$.

Step 5: Check for convergence or the maximum iteration. If the criterion is not satisfied, then set

$$
\Theta^{m}=\Theta^{m+1} \text {, and return to step } 1 \text {. }
$$

When the criterion is satisfied, we define the estimated parameters as $\Theta^{*}=\left(\pi^{*}, \theta^{*}\right)$.

\section{B. Algorithm for Proposed Image Segmentation Model}

When the parameters have been estimated, the next step is to determine to which class the pixel $i$ should be assigned. Based on the proposed model, the algorithm can be summarized as follow.

Step 1: Calculate function $f$

$$
P\left(e_{i k}=1 \mid x_{i}\right)=\frac{\pi_{i k}^{*} P\left(x_{i} \mid \theta_{k}^{*}\right)}{\sum_{d=1}^{K} \pi_{i d}^{*} P\left(x_{i} \mid \theta_{d}^{*}\right)}
$$

Step 2 : Calculate function $F$

$$
P^{*}\left(e_{i k}=1 \mid x_{i}\right)=\sum_{j \in N(i)} \frac{h(i, j)}{Z_{i}} P\left(e_{j k}=1 \mid x_{j}\right)
$$


Step 3 : Calculate function $g$

$$
e_{i k}= \begin{cases}1 & \text { if } k=\arg \max _{t} P^{*}\left(e_{i t}=1 \mid x_{i}\right) \\ 0 & \text { if otherwise }\end{cases}
$$

\section{EXPERIMENTS}

In this section, we provide experimental results on synthetic and real-world images for evaluating our proposed algorithm.

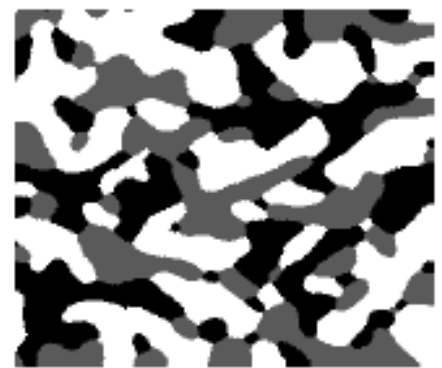

(a)

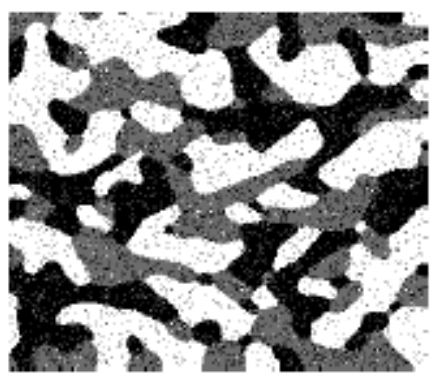

(d)

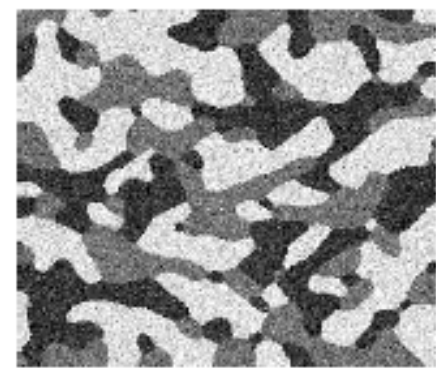

(b)

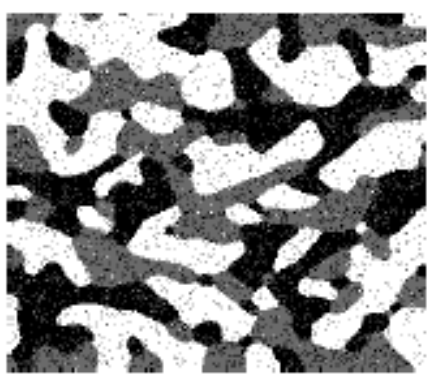

(e)

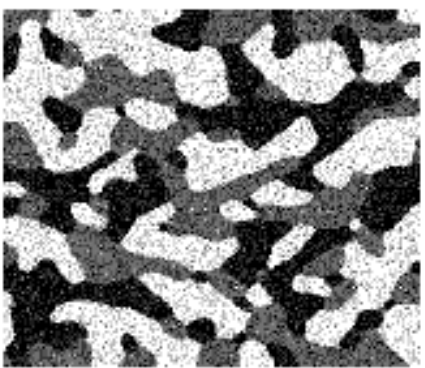

(c)

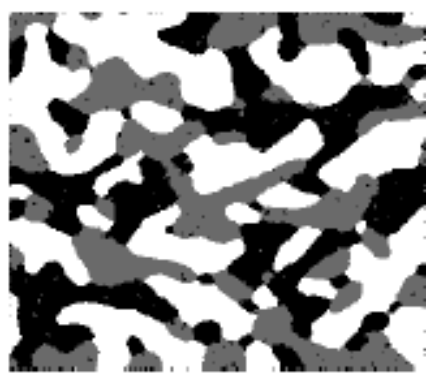

(f)

Fig. 3. Experiment of synthetic image. (a) Original image. (b) Corrupted original image with Gaussian noise(0 mean, 0.02 variance). (c) Standard Mixture Model(MCR=19.24\%). (d) SVFMM (MCR=10.18\%). (e) CA-SVFMM (MCR=9.58\%). (f) Proposed Method (MCR=6.86\%).

\section{A. Parameters estimation}

The algorithm has three kinds of parameters, the number of components $\mathrm{K}$, the parameters of prior distribution $\pi$, and the parameters of all the components $\theta$. In this work we assume that the number of components $\mathrm{K}$ is given to us for a particular image. The choice of the parameters affect the quality of image segmentation directly. In this section, we will estimate the parameters of standard Gaussian mixture model(termed GMM), SVFMM, the extension of mixture model(termed EMM), the class-adaptive SVFMM(termed CA-SVFMM), and the proposed model. We compare the efficiency of the proposed model with the others.

We use a synthetic image (256 x 256 image resolution) similar to the one used in [17], [20] (see Fig.3(a)). The simulated image has three classes $(\mathrm{K}=3)$ sampled from an MRF model using the 
Signal \& Image Processing : An International Journal (SIPIJ) Vol.6, No.4, August 2015

Gibbs sampler [18]. The gray levels for the three classes are 55, 115 and 225 respectively. Fig.3(b) shows the same image with added Gaussian noise (mean=0, variance $=0.02$ ).

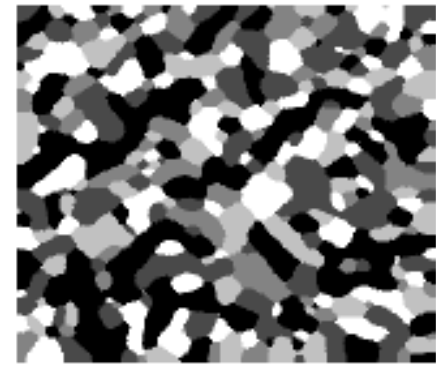

(a)

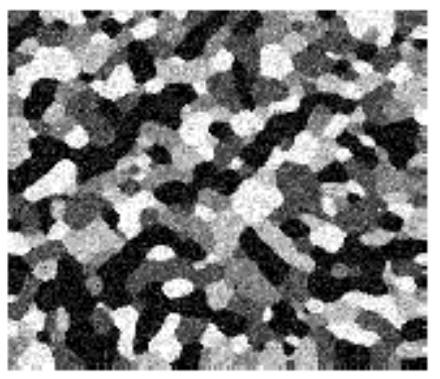

(d)

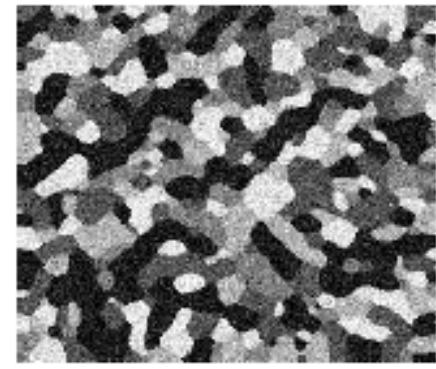

(b)

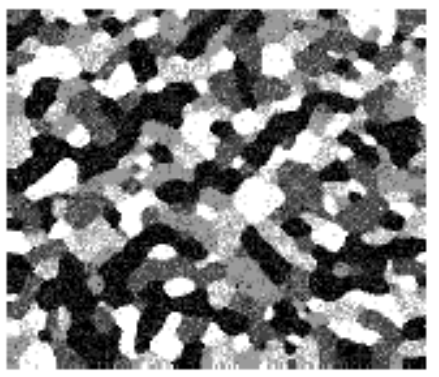

(e)

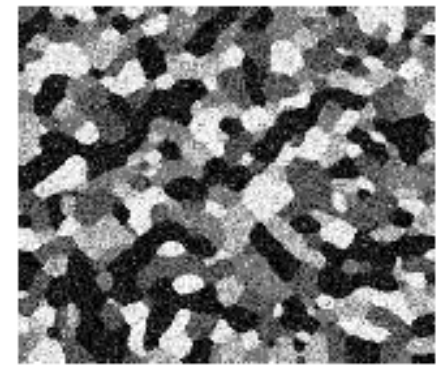

(c)

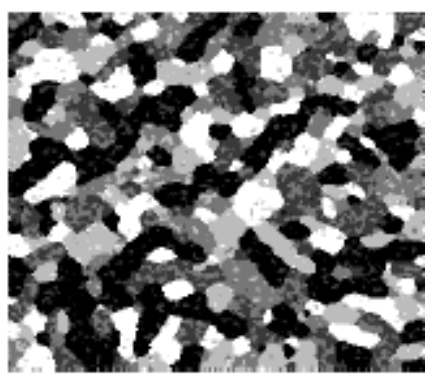

(f)

Fig. 4. Experiment on five-class synthetic image. (a) Original image. (b) Corrupted original image with Gaussian noise (0 mean, 0.01 variance). (c) Standard Mixture Model(MCR=48.10\%). (d) SVFMM $(\mathrm{MCR}=34.31 \%)$. (e) CASVFMM (MCR=31.42\%). (f) Proposed Method $(\mathrm{MCR}=26.23 \%)$.

We use the EM-based algorithm for estimating the parameters of standard GMM, EMM, SVFMM, CA-SVFMM, and our proposed model. Table.I shows the estimated parameters of Fig.3(b). From this table, we can see that estimated parameters of the EMM, SVFMM, CASVFMM and our proposed model are equivalent.

The running times for estimating parameters of the five model are reported in Table.II. The computer used is a PC with Intel Core i 3 CPU 3.07GHz and $8.00 \mathrm{G}$ RAM. From this table, we can see that the standard GMM run fastest. But its parameters are quiet worse than the others. The running time of CA-SVFMM is the slowest.

\section{B. Synthetic Images}

We illustrate the performance of our proposed model with references for synthetic image segmentation. Firstly, we show a comparison of the standard GMM, EMM, SVFMM, CASVFMM and our proposed model for the above three-class image segmentation. We use the misclassification ratio(MCR) [17] to measure the segmentation accuracy, which is defined as

$$
M C R=\frac{\text { number of misclassified pixels }}{\text { total number of pixels }}
$$


Signal \& Image Processing : An International Journal (SIPIJ) Vol.6, No.4, August 2015

Fig.3(a) shows original synthetic image and Fig.3(b) shows the original image with added Gaussian noise (mean=0, variance=0.02). Fig.3(c)-(g) show the segmentation results obtained by standard GMM, EMM, SVFMM, CA-SVFMM and our proposed model. Compared with these five algorithm, it is easy to see that our algorithm obtains the best results. The results obtained with varying levels of noise are presented in Table III. As can be seen, the proposed method has a lower MCR compared with the other methods.

In the second experiment, we illustrate our algorithm on a five-class synthetic image. We use a simulated image $(256 \times 256$ image resolution) similar to the above three-class image(see Fig.4(a)). The gray levels for the five classes are 24, 83, 130, 180 and 230 respectively. In Fig.4(b) we show the image after adding Gaussian noise with 0 mean, 0.02 variance. Fig.4(c)-(g) show the segmentation results obtained by standard GMM, EMM, SVFMM, CA-SVFMM and our proposed model, respectively. Among these methods, the proposed method classify the image with lowest MCR. Moreover, as compared with the other methods, the result obtained by employing our method, as shown in Fig.4(g), demonstrates a higher degree of robustness with respect to the given level of noise.

Form the above examples we can conclude here that the segmentation results of the proposed model are significant quantitative and with the higher degree of robustness with respect to noise

\section{Real World Images}

We have also evaluated and compared the proposed algorithms for the segmentation of RGB natural images. The real world images are obtained from the Berkeley's image segmentation database [29]. This database consists a set of natural images and their ground truth segmentation results provided by different individuals. An error measure (Global Consistency Error) [29] has been defined to quantify the consistency between different segmentations. Global Consistency Error(GCE) produces a real-valued output in the range $[0 ; 1]$, where zero signifies no error. In this paper, we use the GCE to evaluate the performance of our proposed algorithm. Furthermore, we use the probabilistic Rand (PR) index [30] to obtain the comparisons of segmentations of the Berkeley database. It contains values in the range [0,1], with values closer to 1 indicating a good result.

In the first experiment, we tried to segment a color image (482 7321 image resolution) shown Fig.5(a) into three classes: the blue sky, the white church wall and the red cupola. Images in Fig.5(b), (c), (d), (e) and (f) show the results obtained by using the standard mixture model (MM), the extension of MM, SVFMM, CA-SVFMM and our proposed model, respectively. As can be seen, the proposed method outperforms other methods with a higher PR. Also the result of segmentation of our proposed method is quite good. We can observe that the white church wall are slightly better preserved by our method. Furthermore, the bottom right red window and the two crosses are more accurately extracted by our method.

In order to further test the accuracy and determine the efficiency of the proposed method, we do experiment on another real world image. Fig.6(a) shows a color image (321 781 image resolution). We tried to segment the image into three classes. Images in Fig.6(b) (c), (d), (e) and (f) show the results obtained by using the standard MM, the extension of MM, SVFMM, CASVFMM and our proposed model, respectively. Compared with these methods, the proposed method outperforms other methods with a higher PR and a lower GCE. The image shown in 
Signal \& Image Processing : An International Journal (SIPIJ) Vol.6, No.4, August 2015

Fig.6(g) is obtained by corrupting the original image in Fig.6(a) with Gaussian noise (0 mean, 0.01 variance). Fig.6(h) (i), (j), (k) and (l) present the segmentation results obtained by employing the standard MM, the extension of MM, SVFMM, CA-SVFMM and our proposed model, respectively. From visual inspection of the results, our proposed method demonstrates a better performance compared to the other methods. Moreover, the result of the proposed method is with a higher PR and a lower GCE.

In the final experiment, a set of real world images were used to evaluate the performance of the proposed method against standard MM, extension of MM, SVFMM and CA-SVFMM methods. Table IV presents the cumulative results obtained for all methods, for the given set of images. As can be easily seen, the proposed method outperforms the other methods with a higher PR.

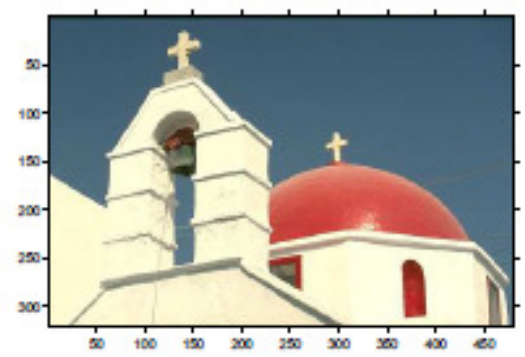

(a)

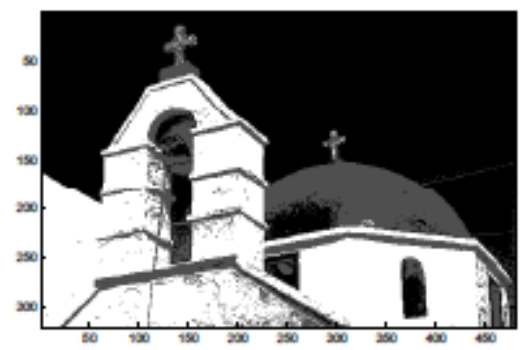

(b)

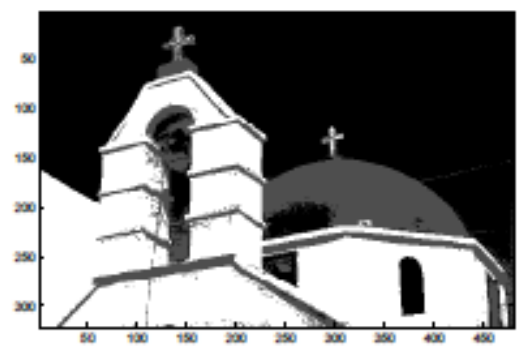

(d)

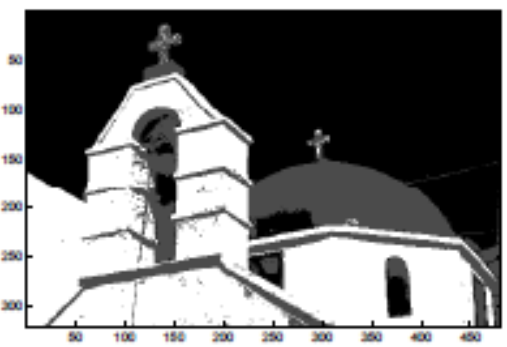

(c)

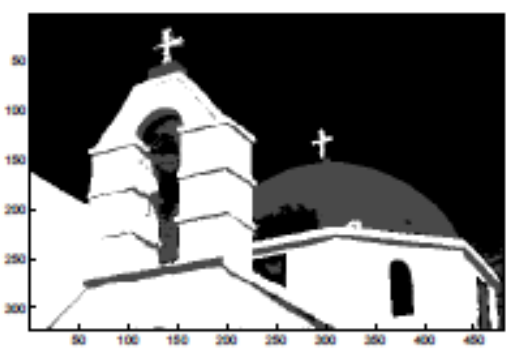

(e)

Fig. 5. Experiment on color image. (a) Original image. (b) Standard Mixture Model ( $\mathrm{PR}=0.830$ ). (c) SVFMM ((PR=0.836). (d) CA-SVFMM ( $\mathrm{PR}=0.838)$. (e) Proposed Method ( $\mathrm{PR}=0.841)$. 
Signal \& Image Processing : An International Journal (SIPIJ) Vol.6, No.4, August 2015

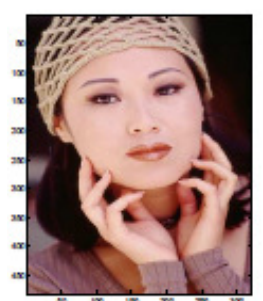

(a)

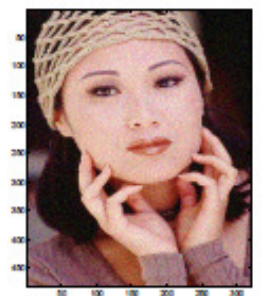

(f)

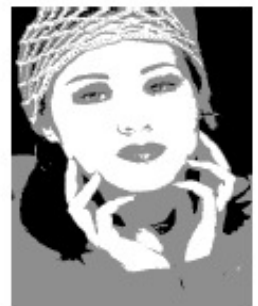

(b)

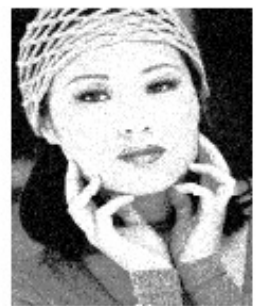

$(\mathrm{g})$

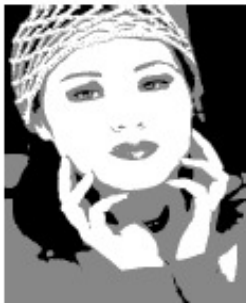

(c)

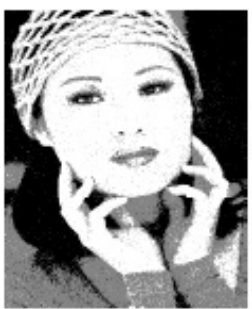

(h)

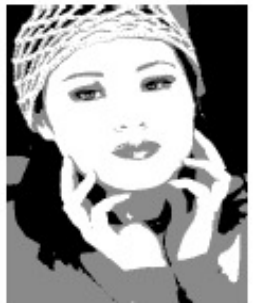

(d)

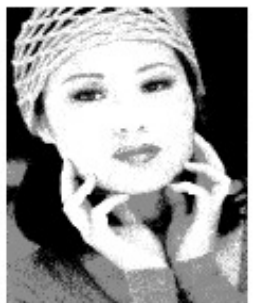

(i)

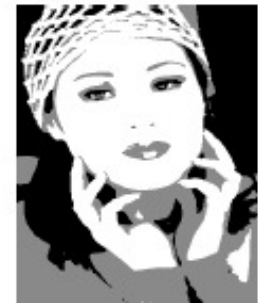

(e)

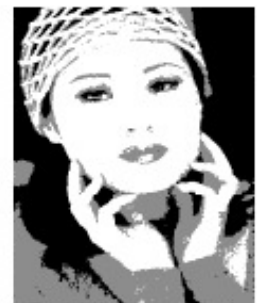

(j)

Fig. 6. Experiment on color image. (a) Original image and its three-class segmentation by (b) $\mathrm{MM}$ $(\mathrm{PR}=0.693, \mathrm{GCE}=0.286), \quad(\mathrm{c}) \mathrm{SVFMM} \quad(\mathrm{PR}=0.713, \mathrm{GCE}=0.267), \quad$ (d) CASVFMM (PR=0.716, $\mathrm{GCE}=0.255$ ), (e) Proposed Method ( $\mathrm{PR}=0.719, \mathrm{GCE}=0.245$ ). (f) Corrupted original image with Gaussian noise (0 mean, 0.01 variance) and its threeclass segmentation by (g) MM ( $\mathrm{PR}=0.697, \mathrm{GCE}=0.342)$, (h) SVFMM (PR=0.709, GCE=0.302), (i) CASVFMM ( $\mathrm{PR}=0.711, \mathrm{GCE}=0.287)$, (j) Proposed Method $(\mathrm{PR}=0.716, \mathrm{GCE}=0.266)$.

\section{CONCLUSION}

In this paper, we have presented a spatially constrained probabilistic field theory-based model for image segmentation. The proposed model postulates that the whole pixels and some unknown parameters form a field and the label of a particular pixel is a compound function of its features, its neighbors' features and some parameters. To estimate the parameters, we have proposed a novel approach to incorporate the spatial relationships between neighboring pixels into the mixture model. The proposed method has been tested with many synthetic and real world images, the experimental results demonstrate the excellent performance of the proposed model in segmenting the images.

\section{REFERENCES}

[1] J. Freixenet, X. Munoz, D. Raba, J. Marti, and X. Cufi, "Yet another survey on image segmentation: region and boundary information integration," 7th European Conference on Computer Vision, pp. 508-422, 2002.

[2] N. Pal and S. Pal, "A review of image segmentation techniques," Pattern Recognition, vol. 26, pp. 1277-1294, 1993.

[3] M. Kass, A. Witikin, and D. Terzopoulos, "Snakes: Active contour models," Int. J. Comput. Vis., vol. 1, pp. 321-331,1988.

[4] C. Xu and J. Prince, "Snakes, shapes, and gradient vector flow," IEEE Trans. Image Process., vol. 7, no. 3, pp. 359-369,1998.

[5] B. Li and S. Acton, "Active contour external force using vector field convolution for image segmentation,” IEEE Trans Image Process., vol. 16, pp. 2096-2106, 2007. 
Signal \& Image Processing : An International Journal (SIPIJ) Vol.6, No.4, August 2015

[6] G. Sundaramoorthi, A. Yezzi, and A. Mennucci, "Sobolev active contours," International Journal of Computer Vision, vol. 73, pp. 109-120, 2005.

[7] J. Shi and J. Malik, "Normalized cuts and image segmentation," IEEE Trans. Pattern Anal. Mach. Intell., vol. 22, no. 8, pp. 888-905, 2000.

[8] D. Comaniciu and P. Meer, "Mean shift: A robust approach toward feature space analysis," IEEE Trans. Pattern Anal. Mach. Intell., vol. 24, no. 5, pp. 603-619, 2002.

[9] R. Xu and I. D. Wunsch, "Survey of clustering algorithms," IEEE Trans. Neural Netw., vol. 16, no. 3, pp. 645-678, 2005.

[10] G. McLachlan, "Finite mixture models," Hoboken, NJ: Wiley, 2000.

[11] F. Picard, "An introduction to mixture model," Statistics for Systems biology Research Report No.7, 2007.

[12] A. Dempster, N. Laird, and D. Rubin, "Maximum likelihood from incomplete data via the EM algorithm," Journal of the Royal Statistical Society.Series B, vol. 39, no. 1, pp. 1-38, 1977.

[13] R. Neal and G. Hinton, "A view of the EM algorithm that justifies incremental, sparse, and other variants," in Learning in Graphical Models, M.Jordan, Ed.Kluwer Academic Publishers, pp. 355-368, 1998.

[14] K. Blekas, D. I. Fotiadis, and A. Likas, "Greedy mixture learning for multiple motif discovery in biological sequences," Bioinformatics, vol. 19, no. 5, pp. 607-617, 2003.

[15] H. Greenspan, G. Dvir, and Y. Rubner, "Context-dependent segmentation and matching in image databases,", Comput. Vis. Image Understand., vol. 93, no. 1, pp. 86-109, 2004.

[16] S. Sanjay-Gopal and T.J.Hebert, "Bayesian pixel classification using spatially variant finite mixture and the generalized EM alogirhm," IEEE Trans. Image Process., vol. 7, no. 7, pp. 1014-1028, 1998.

[17] Y. Zhang, M. Brady, and S. Smith, "Segmentation of brain MR images through a hidden markov random field model and the epectation-maximiztion algorithm," IEEE Trans. Med. Imag., vol. 20, no. 1, pp. 45-57, 2001.

[18] S. Geman and D. Geman, "Stochastic relaxation, gibbs distributions, and the bayesian restoration of images," IEEE Trans. Pattern Anal. Mach. Intell., vol. 6, pp. 721-741, 1984.

[19] S. Z. Li, "Markov random field modeling in computer vision," London,UK: Springer-Verlag, 2001.

[20] K.Blekas, A.Likas, N.P.Galatsanos, and I.E.Lagaris, "A spatially constrained mixture model for image segmentation,” IEEE Trans. Neural Netw., vol. 16, no. 2, pp. 494-498, 2005.

[21] A. Diplaros, N. Vlassis, and T. Gevers, "An spatilly constrained generative mode and an em algorithml for image segmentation," IEEE Trans. Neural Netw., vol. 18, no. 3, pp. 798-808, 2007.

[22] M. Woolrich, T. Behrens, C. Beckmann, and S. Smith, "Mixture models with adaptive spatial regularization for segmentation with an application to fMRI data," IEEE Trans. Med. Imag., vol. 24, no. 1, pp. 1-11, 2005.

[23] C. Nikou, N. P. Galatsanos, and A. Likas, "A class-adaptive spatially variant mixture model for image segmentation,” IEEE Trans. Image Process., vol. 16, no. 4, pp. 1121-1130, 2007.

[24] C. Nikou, A. Likas, and N. P. Galatsanos, "A bayesian framework for image segmentation with spatially varying mixtures," IEEE Trans. Image Process., vol. 19, no. 9, pp. 2278-2289, 2010.

[25] T. M. Nguyen, Q. Wu, and S. Ahuja, "An extension of the standard mixture model for image segmentation,” IEEE Trans. Neural Netw., vol. 21, no. 8, pp. 1326-1338, 2010.

[26] J. L. Marroquin, E. A. Santana, and S. Botello, "Hidden markov measure field models for image segmentation," IEEE Trans. Pattern Anal. Mach. Intell., vol. 15, no. 1, pp. 1380-1387, 2003.

[27] K. Lewin, "Resolving social conflicts; selected papers on group dynamics," Gertrude W. Lewin (ed.) New York: Harper and Row, 1948.

[28] — - "Field theory in social science; selected theoretical papers," D. Cartwright (ed.). New York: Harper and Row., 1951.

[29] D. Martin, C. Fowlkes, D. Tal, and J. Malik, "A database of human segmented natural imaes and its application to evaluation segmentation algorithms and mesauring ecological statistics," in Proc. Int. Conf. Comput. Vision, pp. 416-423, jul.2001.

[30] C. P. R. Unnikrishnan and M. Hebert, "A measure for objective evaluation of image segmentation algorithms,", in Proc. IEEE Conf. Computer Vision and Pattern Recognition, vol. 3, pp. 34-41, Jnn. 2005. 